\title{
Wissenschaft in der Entrepreneurial University - feminisiert und abgewertet?
}

In der universitären Wissenschaft sind so viele Frauen zu finden wie nie zuvor. Gleichzeitig befindet sie sich jedoch in einem Umbauprozess zur „Entrepreneurial University“, wie die grundlegenden Veränderungen in Richtung betriebswirtschaftlicher, wettbewerblicher Steuerungsprinzipien metaphorisch angesprochen werden. Da Prozesse der Feminisierung und der Abwertung von Arbeit nicht selten Hand in Hand gehen, verfolgt der Artikel diese Spur. Gezeigt wird, dass sich eine solche Tendenz durchaus erkennen lässt, die Situation aber nicht eindeutig ist.

BRIGITTE AULENBACHER, KRISTINA BINNER, BIRGIT RIEGRAF, LENA WEBER

\section{Einleitung}

Auf die Frage danach, was die Ausgestaltung von Beschäftigungsverhältnissen und die Verrichtung von Arbeit durch Frauen oder Männer miteinander verbindet, geben die Professionssoziologie und die Geschlechterforschung, wie $A n$ gelika Wetterer (2002) rekonstruiert, eine eindeutige Antwort: Zwar geht die gesellschaftliche Abwertung von Arbeiten, Berufen oder Professionen nicht unbedingt mit veränderten Zuweisungen nach Geschlecht einher, aber da, wo dies geschieht, verbindet sich Abwertung in aller Regel mit Feminisierung. Hingegen vollzieht sich Maskulinisierung gerne in Einklang mit Aufwertung. Diese Gleichgerichtetheit von Maskulinisierung/Aufwertung und Feminisierung/Abwertung ist nicht zwangsläufig. Sie wird, so zeigt Wetterer an zahlreichen historischen und zeitgenössischen Beispielen, in gesellschaftlichen Auseinandersetzungen und Aushandlungen etwa um die Ausgestaltung von Beschäftigungsverhältnissen und -bedingungen, von Arbeitsinhalten, des jeweiligen Berufs- und Professionsverständnisses mal mehr, mal weniger intendiert immer wieder neu hervorgebracht.

Im Wissenschaftssystem steigt der Anteil von Frauen in den letzten Jahren kontinuierlich an. Dieser Anstieg kann als Erfolgsgeschichte der nachwirkenden Bildungsreform der 1970er Jahre und der seitherigen Gleichstellungspolitiken von der frühen Frauenförderung über Gender Main- streaming bis zum Diversity Management erzählt werden (Meuser/Riegraf 2010). Durch die Brille der zitierten professionssoziologischen Geschlechterforschung betrachtet, ist allerdings zu bedenken: Er vollzieht sich zu einem Zeitpunkt, zu dem die Wissenschaft die Exklusivität, das Ansehen und die Bedeutung, die mit der Humboldt'schen Ordinarienuniversität noch verbunden waren, einbüßt und mit der „Entrepreneurial University“ (in Anlehung an Clark 1998) eine ganz neue Weichenstellung erfährt. Folgende Fragen drängen sich auf: Steigen Frauen in die universitäre Wissenschaft ein und in ihr auf, während deren gesellschaftlicher Stellenwert insgesamt sinkt? Sind die Tätigkeitsbereiche, in denen sich Frauen wiederfinden, diejenigen mit der geringsten Reputation innerhalb des Feldes? Wo und wie bilden sich Privilegien heraus, wo und wie werden die Einzelnen in ihrer wissenschaftlichen Arbeit blockiert? Oder zeigen sich Risse in der Homologie von Männlichkeit/Aufwertung und Weiblichkeit/Abwertung?

In Abschnitt 2 werden in kursorischen Beschreibungen vergeschlechtlichte Entwicklungen beleuchtet, die der Herausbildung der Entrepreneurial University vorgelagert sind und teilweise fortgeführt, teilweise verändert, teilweise zur Disposition gestellt werden. In Abschnitt 3 stehen der Wandel der Beschäftigungsverhältnisse im Zentrum, wie er sich im Umbau der Universitäten zur Entrepreneurial University vollzieht, sowie seine Verbindung mit der Gleich- und Ungleichstellung der Geschlechter. In einem vierten Schritt werden erste Antworten auf die genannten Fragen gegeben (4.). 


\section{Wissenschaft und Geschlecht im Rückblick}

Die Herausbildung der Entrepreneurial University wird mit einiger Berechtigung als Bedrohung oder gar Ende der mit dem Namen Humboldt verbundenen Organisation von Wissenschaft interpretiert. Die Einführung betriebs- und marktwirtschaftlicher Organisations- und Steuerungsmechanismen in die Wissenschaftsadministration geht mit der Zurückdrängung des Kollegialmodells der Selbstverwaltung einher. Die Verstärkung der Drittmittelforschung tangiert die Freiheit der Forschung. Die Architektur der Bachelor- und Masterstudiengänge lässt die Einheit von Forschung und Lehre wie damit das Selbststudium als Säule des wissenschaftlichen Lernprozesses fraglich werden (Schimank 2005).

Weniger gerät ein weiteres bedeutendes Moment in den Blick: Das mit dem Humboldt'schen Ideal verbundene professionelle Verständnis, die Berufung zur Wissenschaft, wie sie Max Weber (1994) in seinem berühmten Aufsatz „Wissenschaft als Beruf" aufnimmt. Weber wie zuvor auch Humboldt verbinden damit das ausschließliche Streben nach und die Verpflichtung auf Erkenntnisgewinn. Sie zeichnen das noch ältere Bild des in Einsamkeit und Freiheit forschenden Gelehrten nach, der sich selbst und seine politischen, ökonomischen und sozialen Anschauungen zugunsten des wissenschaftlichen Erkenntniswillens zurücknimmt (auch Haraway 1996). Dabei wird nicht in Abrede gestellt, dass subjektive Präferenzen und Interessen die Ausrichtung von Forschung prägen; Weber erkennt sie sogar ausdrücklich als ideenstiftend und damit auch richtungsweisend an. Jedoch dürfe Wissenschaft nicht in den Dienst anderer Belange als des Erkenntnisgewinns gestellt werden. Die Politik beispielsweise habe „im Hörsaal nichts zu suchen" (Weber 1994, S. 28).

Dass diese tradierte Vorstellung des zur Wissenschaft Berufenen von gesellschaftlichen Arbeitsteilungen nach Geschlecht unterfüttert ist, darauf machte in erster Linie die Geschlechterforschung aufmerksam. Sigrid Metz-Göckel (2009) zeigt, wie „Wissenschaft als Lebensform“ (Mittelstraß 1982) betrieben wird, um das Privileg ausschöpfen zu können, sich einer dem anerkannten Anspruch nach rein erkenntnisorientierten Arbeit zu widmen und ganz in den Dienst der Sache zu stellen. Die Konstellationen, die sich dabei herausbildeten, sahen für Frauen und Männer historisch höchst verschieden aus. Während der Berufene seine Freistellung für die Arbeit am Erkenntnisgewinn nicht selten seiner Lebensgefährtin oder Ehefrau verdankte und Frauen über lange Zeit aus der Wissenschaft gänzlich ausgeschlossen waren (Noble 1992), hatte die Berufene, endlich in der Wissenschaft angelangt, für sich selbst und gegebenenfalls noch für andere zu sorgen bzw. wurde zumindest nicht in gleicher Weise umsorgt. Mit aktuellen arbeitssoziologischen Begriffen ließe sich sagen, dass Wissenschaft immer schon entgrenzte und subjektivierte Arbeit war, die andere Bereiche des Lebens überlagerte (Aulenbacher 2010).
Arbeit in der Wissenschaft war immer auch, um einen weiteren zeitgenössischen Begriff aufzunehmen, prekär, was in Max Webers Rede vom „Hazard“ (1994, S. 7) zum Ausdruck kommt. Die frühen Gelehrten waren nicht selten ganz ohne oder ohne festes Einkommen, was sich bis zu den heutigen Privatdozenturen erhalten hat (Matthies 2005, S. 159ff.), wie beispielsweise die Initiative Berliner Privatdozenten immer wieder kritisiert (BIP 2012) und womit sie eher die Ausnahme bildet hinsichtlich der weitgehend kritik- und kampflosen Akzeptanz von Existenzrisiken um der Berufung willen (Dörre/Neis 2008). In der tradierten wissenschaftstypischen unsicheren Laufbahnkonstruktion verbanden sich professioneller Erfolg, bemessen an der fachlichen Reputation, mit wirtschaftlicher Sicherheit vor allem dann, wenn eine Berufung auf eine autonome Professur oder, im Sinne Humboldts, als Ordinarius erfolgt war, wobei Männer nahezu unter sich blieben.

Nicht zuletzt die Proteste der 1968er Bewegung, die in die Reformbestrebungen der 1970er Jahre übergingen, führten zur ersten großen Wende der Nachkriegszeit in der ursprünglichen institutionellen Einbindung und organisationalen Ausgestaltung der Hochschulen und im professionellen Selbstverständnis von Wissenschaft. Auch die neue Indienstnahme der Hochschulen für die Ausbildung und Entwicklung des, wie es jetzt hieß, Humankapitals für andere als wissenschaftliche Bereiche trug dazu bei. Die elitären Ordinarienuniversitäten wurden über Reformen der Hochschulverfassung demokratisiert und die universitäre Ausbildung wurde für breitere Schichten der Bevölkerung und dabei mithilfe der ersten Frauenförderinitiativen in neuem Maße und gezielt für Frauen geöffnet (Sambale et al. 2008). Die Humboldt'sche Idee der Freiheit und Einheit von Forschung und Lehre blieb in dieser Phase noch erhalten.

Gegenüber der vorherigen Ordinarien- erfuhr die Reformuniversität durch den Verlust ihres elitären Charakters jedoch sukzessive eine Abwertung in der gesellschaftlichen Anerkennung und in der Selbstwahrnehmung von Professoren. Das Zitat des Soziologen und offensichtlichen Reformgegners Hartmut Esser (2002, S. 20) bringt dies beispielhaft für sein (und unser) Fach zum Ausdruck. Soziologie entwickle sich „[...] von einem einstmals sehr angesehenen Sammelpunkt" begabter Lehrender und Studierender zu einem Sammelbecken mäßig Qualifizierter und Interessierter, was einen „baldigen Absturz im öffentlichen Ansehen der Soziologie" nach sich gezogen habe. Die Öffnung der Hochschulen wird hier nahtlos mit einem Bedeutungsverlust und einer Abwertung von Wissenschaft verschränkt. Zurückhaltender skizziert Richard Münch (2009) die Situation, wenn er feststellt, dass Reformuniversitäten die Strukturreformen anders für sich zu nutzen wussten, während Traditionsuniversitäten in den Hintergrund traten.

Mit der gegenwärtigen Reorganisation der Wissenschaft nach dem Format der Entrepreneurial University ist erneut ein grundlegender Wandel des Wissenschaftssystems zu verzeichnen, der die institutionelle Einbettung, die organi- 
sationale Ausgestaltung insgesamt sowie das Professionsverständnis nicht unberührt lässt. Dieser Prozess verschränkt sich in neuer Weise mit Gleichstellungskonzepten. Wie ist es vor diesem Hintergrund nun mit den skizzierten Homologien im Umbau der Universitäten zur Entrepreneurial University bestellt?

\section{Auf dem Weg in die Entrepreneurial University}

Mit dem Aufbau der Entrepreneurial University entlang der Prinzipien des New Public Management (NPM) erreichen Ökonomisierungsprozesse, die in den letzten Dekaden im öffentlichen Sektor in nahezu allen OECD-Ländern über die Einführung neuer wettbewerbsorientierter, betriebswirtschaftlicher Organisations- und Steuerungsmechanismen zu beobachten sind, auch das Wissenschaftssystem (Münch 2009). Bisherige staatlich-bürokratische Detailregulierungen (wie bei der Haushaltsplanung) werden zunehmend durch allgemeine Vorgaben (wie das Erreichen von Exzellenz, Internationalität oder Innovation, deren Übersetzung und Konkretisierung einzelnen Hochschulen überlassen wird) und outputorientierte Kontextsteuerung ersetzt (Riegraf 2007a, b; 2009). Wenngleich das bisherige Wissenschaftssystem keineswegs ein interessenfreier Raum war, so ist durch die Einführung der NPM-Instrumente und das Bologna-Ziel der Anwendungsorientierung, wie es mit den neuen Studiengängen als „Employability“ zum Tragen kommt, die Bindung an außerwissenschaftliche Kriterien und damit auch außerwissenschaftliche Interessen enger als bislang gespannt worden (Teichler 2008). Diese Entwicklung ist in Bezug auf den Zugang von Frauen zur Wissenschaft und die Ausgestaltung der Beschäftigungsverhältnisse, die als ein Indikator für das Ansehen des Berufs- und Tätigkeitsfeldes gelten können, zwiespältig und wirkt sich auf das Ansehen der Profession aus. Sie verbindet sich zudem in neuer Weise mit Gleichstellungskonzepten und -politiken, die ganz anderen Ursprungs sind, etwa mit dem Gender Mainstreaming, das im Zuge der EU-Richtlinien umgesetzt werden muss, oder dem der Privatwirtschaft entlehnten Diversity Management (Meuser/Riegraf 2010).

\subsection{New Public Management und Geschlechterpolitiken}

Im Rahmen des economic und organisational shift (Musselin 2007; Schimank 2005), der staatlich initiierten und sanktionierten Wettbewerbe der Hochschulen um finanzielle Ressourcen, wird Gleichstellung in der Wissenschaft in ganz neuer Weise thematisiert und thematisierbar. Nicht zuletzt aufgrund der Kritik ausländischer Gutachterinnen und Gutachter wurden beispielsweise mit der Einführung des neuen Forschungsförderungsprogramms der deutschen
„Exzellenz-Initiative“ Gleichstellungskonzepte Bestandteil bei der Vergabe des Titels „Exzellenzuniversität“ (auch Hartmann 2010), was an einigen Universitäten durchaus zu innovativen Gleichstellungskonzepten führte. Auch in universitären Entwicklungsplänen und Ziel- und Leistungsvereinbarungen wurden Gleichstellungsmaßnahmen verankert (Becker et al. 2010). Österreich finanzierte von Ministeriumsseite beispielsweise zur Habilitationsförderung Postdoc -Stellen für Frauen und vergab diese an die Universität. Das Professorinnen-Programm von Bund und Ländern in Deutschland zielte darauf ab, über finanzielle Anreize den Frauenanteil an Hochschulen zu erhöhen. Etwa die Hälfte der deutschen Hochschulen bewarb sich, wovon drei Viertel aus diesem Programm gefördert und 260 (statt ursprünglich 200 geplante) Professorinnenstellen vergeben wurden. Allerdings stellt sich die Frage, wie dauerhaft solche Veränderungen sind, wenn sie von wiederholten Wettbewerbssituationen abhängen.

Die Gleichstellungsstrategien des Gender Mainstreaming und des Diversity Managements, die inzwischen an den Hochschulen implementiert werden, weisen anders als die früheren, der Frauenbewegung entstammenden Frauenförderkonzepte eine hohe Anschlussfähigkeit an den economic shift beziehungsweise an die organisationalen Logiken des New Public Management auf. Sie sind rechtlich unverbindlich und unbestimmt und können wie im Falle des Gender Mainstreaming aufgrund ihrer inhaltlichen Unbestimmtheit an betriebswirtschaftliche Instrumente wie das Controlling gekoppelt werden. Oder sie legen wie im Falle des Managing Diversity als Human-Resource-Ansatz unterschiedliche Personalressourcen als Produktivitätspotenziale aus (Meuser/Riegraf 2010). Diese Gleichstellungskonzepte vollziehen den Übergang von verwaltungs- zu markteffizienten Organisations- und Steuerungspolitiken mit. Da keine einheitlichen, verbindlichen und flächendeckenden Konzepte existieren, wird sich ihre Wirksamkeit jedoch vor allem auf der Ebene der Beschäftigungsverhältnisse erst noch erweisen müssen.

\subsection{Die Trennung von Forschung und Lehre und die Arbeitsteilung der Geschlechter}

Die Entrepreneurial University beinhaltet eine Neuorganisation von Forschung und Lehre, unterfüttert von geschlechtsbasierten Zuweisungen von Beschäftigungsverhältnissen und -bedingungen. Jenseits individueller Verdienste werden Forschung und Lehre sehr unterschiedlich honoriert. Für den britischen Kontext belegen Studien, dass sich Frauen vermehrt auf lehrintensiven Stellen finden (Leontowitsch/Vaquéz-Cupeiro 2003), was ihnen im Weiteren den Zutritt zu forschungsträchtigen „premier league-“ jobs erschwert (Thomas/Davies 2002). Robyn Thomas und Annette Davis sprechen gar von der Tendenz eines geschlechterbezogenen „dual labour market model“ (ebd., S. 386) im britischen Wissenschaftsbetrieb, in denen Männer eher forschungsintensive und reputationsträchtige 
Stellen und Frauen weniger reputationsträchtige Stellen in der Lehre besetzen. Darüber, wie die Geschlechter im deutschen Hochschulraum sich auf Lehr- und Forschungsstellen segregieren und inwiefern sich somit ungleiche Chancen für eine wissenschaftliche Weiterqualifizierung zeigen, ist bislang wenig bekannt.

Im deutschsprachigen Kontext entstanden in den letzen Jahren neue Personalkategorien, die ausschließlich auf Lehre fokussiert sind und kaum Raum für forschungsorientierte Arbeiten lassen. Österreich führte „Senior Lecturers“ ein, die bis zu 18 Semesterwochenstunden (SWS) in der universitären Lehre tätig sind (DU 2011). Daten, die für das Jahr 2010 vorliegen, untermauern die These, dass Frauen mit $52,4 \%$ in der Personalkategorie leicht dominieren (http:// eportal.bmbwk.gv.at). Deutschland konzipierte die Beschäftigungskategorie „Lehrkräfte für besondere Aufgaben“ mit einem Lehrdeputat von bis zu 16 SWS an Universitäten (mehr als 20 SWS an Fachhochschulen). Verglichen mit anderen Personalkategorien ist der Frauenanteil auf diesen Stellen mit $48 \%$ überproportional hoch (Bloch/Burkhardt 2010, S. 39).

Die neuen lehrintensiven Tätigkeiten stellen, wird eine weitere Wissenschaftskarriere angestrebt, die Anforderung, sich trotz des hohen Lehrdeputats an den vor allem forschungsorientierten professionellen Normen der scientific community zu orientieren und entsprechende Publikationsund Forschungsleistungen vorzulegen. Befinden sich Frauen überproportional auf diesen Stellenformaten, entsteht im Vergleich zur männlichen Konkurrenz eine strukturelle Ungleichheit im Wettbewerb. Forschen und Schreiben ,in the job“ ist hier nicht mehr vorgesehen. Sofern die Lebenssituation es zulässt, kann und muss ein Teil der Arbeit „,contra the job" und im Sinne einer noch weiter entgrenzten Arbeit in der eigentlich erwerbsarbeitsfreien Zeit erfolgen - gleichsam als eine neue Variante von „Wissenschaft als Lebensform“ (Mittelstraß 1982).

Die Einwerbung von Drittmitteln gilt als vermeintlicher „Königsweg“ (Münch 2007) einer verbesserten Wissenschaftsstruktur. Die Anforderungen treffen auf Wissenschaftsfelder, die diesen ungleich nachkommen können. Beispielsweise sind die Geistes- und Sozialwissenschaften, in denen sich Frauen am ehesten finden, vergleichsweise drittmittelschwach und einem stärkeren Umstrukturierungsdruck ausgesetzt (Meier/Schimank 2004). Drittmittelfinanzierte Stellen eröffnen eher Zeit zur wissenschaftlichen Weiterqualifikation, sind von dem Aufwand für Lehre befreit und bieten für die Forschung aussichtsreichere Kontakte (Grühn et al. 2009). In den drittmittelstarken, von Männern dominierten Natur- und Ingenieurwissenschaften trifft der Nachwuchs eher auf diese Stellenkonstellationen. Der Anteil an wissenschaftlichen Mitarbeitern und Mitarbeiterinnen, die durch öffentliche Drittmittel finanziert werden, ist in der Mathematik und den Naturwissenschaften um 28 \% höher als in den Rechts-, Wirtschafts- und Sozialwissenschaften (Statistisches Bundesamt 2011). An österreichischen Universitäten werden drittmittelfinanzierte Stellen von Männern dominiert, 69,7 \% der forschungs- intensiven Vollzeitstellen sind mit Männern besetzt (http:// eportal.bmbwk.gv.at/).

\subsection{Prekarisierung der Beschäftigungs- verhältnisse und Einzug der Frauen}

Gegenwärtig spitzt sich die Unsicherheit von wissenschaftlichen Karrieren (Enders 2003) und Beschäftigungsverhältnissen zu (Matthies 2005), was insbesondere den wissenschaftlichen Mittelbau trifft (Dörre/Neis 2008; Grühn et al. 2009). Österreich setzt über flächendeckende Entwicklungspläne auf eine effiziente und dynamische Gestaltung der Personalstruktur auf allen Beschäftigungsebenen. So wurden Prädoktorandenstellen als Ausbildungsverhältnisse konzipiert, deren Verlängerung nach vier Jahren nicht vorgesehen ist und die nicht in die Stellenpläne der Universitäten integriert sind. Ähnliche Befristungspraxen setzen sich auch im Mittelbau durch, die vormals verbeamteten Stellen sind nun auf sechs Jahre befristet und mit Qualifikationsanforderungen versehen, die beispielweise die Anforderung enthalten können, Auslandsaufenthalte zu absolvieren (BMBWK 2006, S. 52).

In Deutschland gilt eine wissenschaftliche Karriere schon länger als wenig planbar. Insgesamt ist der Mittelbau erneut von abgewerteten, prekären Beschäftigungsverhältnissen betroffen, Wissenschaftlerinnen jedoch stärker im Vergleich zu ihren Kollegen. Während 77 \% der Männer und 80 \% der Frauen an deutschen Universitäten in befristeten Beschäftigungsverhältnissen angestellt sind, zeigen das Beschäftigungsausmaß und die Stellenprofile weitere geschlechterbezogene Segmentationslinien: Im Mittelbau besetzen mit 59 \% weit mehr Frauen als Männer Teilzeitstellen, die lediglich mit 38 \% vertreten sind (Metz-Göckel et al. 2010).

Auch auf professoraler Ebene finden sich Abwertungen durch neue Stellenformate zu einem Zeitpunkt, an dem Frauen zwar noch immer zahlenmäßig gering vertreten sind, aber auch in hohen Positionen in der Wissenschaft ankommen. In Deutschland sind etwa 17 \% der Lehrstühle mit Frauen besetzt, in der höchsten Besoldungsgruppe (C4/ W3) sind es lediglich $13 \%$ (Brugger/Buschle 2010); in Österreich sind Frauen zu $16 \%$ auf Universitätsprofessuren vertreten (BFD 2010). Doch wie „spitze“ sind diese Spitzenpositionen noch angesichts der dienstrechtlichen Reformen? Seit der Einführung der W-Besoldung in Deutschland und mit der zunehmenden Herausbildung der Entrepreneurial University ist die Professur nicht mehr das versprechensreiche Ziel entbehrungsreicher Qualifikationsphasen.

Die W-Besoldung bedeutet gegenüber der C-Besoldung abgesunkene Grundgehälter. Leistungszulagen sind Verhandlungssache (z. B. bei der Einwerbung von Drittmitteln), wobei geschlechterungleiche Verhandlungserfolge vermutet werden. Die leistungsbezogenen unterscheiden sich nochmals nach ruhegehaltsfähigen und nicht ruhegehaltsfähigen Zulagen. Dabei zeigen sich - auch unter Berücksichtigung der Dienstjahre - ebenfalls signifikante geschlechterunglei- 
che Verteilungen (Simons/Hellemacher 2009; Hellemacher 2011). Eine nicht repräsentative Umfrage ergab, dass $19 \%$ der Hochschullehrerinnen in der W-Besoldung ruhegehaltsfähige Zulagen erhalten, während es unter den Hochschullehrern $29 \%$ sind.

In Österreich zeigen sich ebenfalls Abwertungen der Professur. Die Verbeamtung wurde abgeschafft und die Gehälter sind deutlich gesunken (Pasternack 2008). Einzelne Universitäten führten Gehälter mit einem leistungsorientierten Gehaltsbestandteil ein (BMBWK 2006, S. 51). Nach 2002 geschaffene Professorenstellen können auf drei bis fünf Jahre befristet werden. Entfristungen können als Ergebnis einer Evaluierung erfolgen. Mit Einsetzen dieser Beschäftigungspraxis finden sich Frauen vermehrt auf Professuren. Waren im Jahr der Einführung des UG 2002 nur 10 \% Professoren weiblich, erhöhte sich der Frauenanteil bis zum Jahre 2010 auf 19 \% (Statistik Austria 2011). Diese „neu eingetretenen“ Professorinnen wurden allerdings häufig zu anderen Beschäftigungsvoraussetzungen angestellt als ein Großteil ihrer männlichen Kollegen: Zu rund einem Drittel sind sie im Rahmen von befristeten Beschäftigungsverhältnissen beschäftigt, hingegen finden sich nur $17 \%$ der Professoren auf den neu geschaffenen, befristeten Professuren (BMBWK 2006).

\subsection{Exzellenz, Lebensführung und Geschlecht}

Veränderte Kriterien wissenschaftlicher Exzellenz, hohe Drittmittelquoten, umfassende Publikationsoutputs, bruchlose Karriereverläufe und große Mobilitätsbereitschaft markieren gewandelte Vorstellungen von exzellenten Wissenschaftlern. Aber nicht überall, wo der Anspruch auf exzellente Forschung formuliert wird, sind die Voraussetzungen dafür bereitet. Dies zeigen das internationale Gefälle zwischen den Eliteuniversitäten und die sich national durchsetzende Unterscheidung von Forschungs- und Lehruniversitäten (Thomas/Davies 2002) bzw. die Differenzierung zwischen Exzellenz- und Massenuniversität (Hartmann 2010).

Im Zusammenwirken mit den neuen Kriterien wissenschaftlicher Exzellenz kann die private Lebensführung weiterhin karrierehinderlich sein. Noch immer unterscheiden sich die Lebensarrangements von Wissenschaftlerinnen und Wissenschaftlern auf Spitzenpositionen erheblich. Professoren sind in Deutschland häufiger verheiratet (91\%) als Professorinnen (66 \%) und haben im Durchschnitt mehr Kinder $(1,77)$ als Professorinnen $(0,8)$. Hochschullehrerinnen sind häufiger ledig (20\%) im Vergleich zu ihren Kollegen (3 \%) und kinderlos (50 \%) (vgl. Zimmer et al. 2007). Jeweils zwei Drittel der österreichischen und deutschen befragten Wissenschaftler gaben an, dass die Betreuung der Kinder von den Partnerinnen übernommen werde. Im Kontrast dazu sagen nur $8 \%$ der deutschen und $11 \%$ der österreichischen Professorinnen, dass sie von ihren Partnern bei der Kinderbetreuung entlastet werden. Hochschullehrer leben nach wie vor häufiger in traditionellen Paar- arrangements und sind im Vergleich zu ihren Kolleginnen von der Sorge um Kindererziehung und -betreuung eher entlastet (Zimmer et al. 2007; Buchholz 2004). Damit stellen wir abschließend erneut die Frage nach der Feminisierung und Abwertung der Wissenschaft und geben erste Antworten.

\section{Abwertung und Feminisierung der Wissenschaft? Fazit und Ausblick}

War die Humboldt'sche Universitas zugleich eine Ordinarienuniversität, die ihren in Forschung und Lehre autonomen Professoren ein privilegiertes Arbeiten um der wissenschaftlichen Erkenntnis willen erlaubte, so sind diese Freiräume in der Entrepreneurial University und damit in der Phase, in der gezielt um Frauen geworben wird, beschnitten worden. Während von der Ausweitung und Absicherung der Beschäftigungsverhältnisse in der Reformuniversität in erster Linie Wissenschaftler und in zweiter Linie Wissenschaftlerinnen profitierten, so scheint sich in der Entrepreneurial University die Kluft in anderer Weise aufzutun. Die neuen Segmentierungen und Segregationen entlang der Linie Forschung und Lehre vollziehen sich nach Geschlecht. Die Entrepreneurial Science scheint damit eine neue Plattform für Verteilungskämpfe zu bieten, in denen sich Aufwertung und Maskulinisierung der einen Wissenschaftsbereiche neben Abwertung und Feminisierung der anderen Bereiche vollziehen.

Wie betreibt der „new scientific entrepreneur" (Aulenbacher/Riegraf 2010a; Aulenbacher/Riegraf 2010b) „Wissenschaft als Lebensform“ (Mittelstraß 1982)? Zeigt sich, wie Alexandra Rau (2010, S. 411ff.) für die Entgrenzung und Subjektivierung von Arbeit in anderen Bereichen vermutet, auch in der Wissenschaft, im breiten Mittelfeld unterhalb der Exzellenz und oberhalb des Lehrverschleißes, eine Feminisierung der Erwerbsarbeit bei gleichzeitiger Maskulinisierung der Lebensführung und -verhältnisse? Und wie sieht es mit der Homologie von Maskulinisierung/ Aufwertung und Feminisierung/Abwertung aus?

Die Sichtung des Forschungsstandes ergibt kein eindeutiges Bild: Die Rede von der weiteren Feminisierung der Erwerbsarbeit ist plausibel und auch eine homologe Abwertung lässt sich erkennen. Frauen erreichen Positionen, die ihnen bislang verschlossen waren, zu einem historischen Zeitpunkt, zu dem sie nicht mehr mit den Gratifikationen verbunden sind, die vorherigen Generationen und Männern zukamen (Aulenbacher 2010, 2011) - die Entwicklung vom Ordinarius zur befristeten Professur im Angestelltenverhältnis ist das zugespitzte Beispiel dafür. Gleichgerichtete Prozesse der Auf-/Abwertung und der Maskulinisierung/ Feminisierung lassen sich zudem in der auch geschlechtsbasierten Segregation und Hierarchisierung von Forschungs- und Lehrtätigkeiten erkennen. 
Die Rede von der Maskulinisierung der Lebensführung und -verhältnisse hingegen ist fraglich. Die damit verbundene Assoziation, dass nun auch Frauen voll für Erwerbsarbeit verfügbar sind und sein müssen, ist zwar in gewisser Weise nachvollziehbar. Auch mögen Faktoren wie erhöhte Erwartungen an die internationale Mobilität und die Verlagerung steigender Anteile wissenschaftlichen Arbeitens auf den Feierabend dazu beitragen, dass sich das bisherige androzentische Lebensmodell nun verallgemeinert, indem es in neuem Umfang auch Wissenschaftlerinnen erfasst. Dennoch trägt die Rede von einer Maskulinisierung der Lebensführung und -verhältnisse nicht allzu weit.

Zum einen gehörte zur maskulinen Lebensführung unausgesprochen immer schon das Versorgtsein durch andere, wie es für den in Einsamkeit und Freiheit forschenden Gelehrten und sein organisationales Pendant, den autonomen Professor, angesprochen wurde. An diesem Teil maskuliner Lebensführung haben den Daten nach Wissenschaftlerinnen bis heute nicht teil. Zum anderen lässt sich das Engagement der „,neuen Väter“, die es auch in der Wissenschaft gibt (Liebig 2010) und die ebenso wie Generationen von Müttern vor ihnen die entgrenzte, subjektivierte und prekäre Arbeit kritisieren, nicht als Maskulinisierung der Lebensführung im Sinne der Fortschreibung des bisherigen androzentrischen Modells beschreiben.

Nicht nur das Phänomen „neue Väter“ als Wissenschaftler, sondern überhaupt die Frage, wer unter welchen Voraussetzungen und Bedingungen wie Wissenschaft betreibt und wie sich diese Konstellationen für die Geschlechter entwickeln, lässt sich mit Blick allein auf die inneruniversitäre Entwicklung nicht zureichend verfolgen. Es bedarf einer Erweiterung auf die Analyse der alltäglichen und biografischen Arbeitsarrangements von Wissenschaftlern und Wissenschaftlerinnen, wenn beurteilbar werden soll, in welcher Weise und um welchen Preis sich Frauen und Männer in der Wissenschaft engagieren können und wollen. Denn die Anforderungen, die sie in der Entrepreneurial University an sich gestellt sehen, sind nur ein Teil der Belange, die dabei ins Gewicht fallen. Sie sind in Zusammenhang zu sehen mit weiteren Belangen des Lebens und Unterstützungsleistungen, auf die zu ihrer Bewältigung zurückgegriffen wird, sei es in privaten Bezügen, sei es durch den Sozialstaat bzw. in Verbindung damit (vgl. Aulenbacher/Riegraf 2011; Aulenbacher et al. 2010; Binner 2010; Binner et al. 2010). Nicht allein universitäre, sondern auch außeruniversitäre Konstellationen entscheiden mit darüber, inwieweit die neuen Anforderungen wissenschaftlichen Arbeitens, sei es der hohe Publikationsoutput, sei es die internationale Mobilität, erfüllt werden oder ob und in welchem Ausmaß sich Widerstand gegen diese und weitere Neuorientierungen und die forcierte Prekarisierung der Wissenschaftlerexistenz durch die Beschäftigungsformate der Entrepreneurial University regt. In diesem Sinne ist Wissenschaft im Dreieck von Entrepreneurial University, privater Lebensführung und Sozialstaat in den Blick zu nehmen, wenn die Frage danach weitergehend beantwortet werden soll, in welcher Weise die Geschlechter daran teilhaben werden.

\section{LITERATUR}

Aulenbacher, B. (2010): What's New? Der Wandel der Arbeitsgesellschaft geschlechter- und arbeitssoziologisch begriffen, in: Frey, M./Heilmann, A./Lohr, K./Manske, A./Völker, S. (Hrsg.): Perspektiven auf Arbeit und Geschlecht, München/Mehring S. 75-101

Aulenbacher, B. (2011): Frauen, Männer, Prekarität. Vom fordistischen Versprechen auf Wohlstand zur postfordistischen Reproduktionskrise, in: Hammerschmidt, P./Sagebiel, J. (Hrsg.): Die Soziale Frage zu Beginn des 21. Jahrhunderts, München/Neu-UIm, S. 121-136

Aulenbacher, B./Binner, K./Riegraf, B./Weber, L. (2010): „Brot und Rosen“ oder: Der unerhörte Anspruch auf ein gutes Leben innerhalb und außerhalb der Wissenschaft, in: Bauschke-Urban, C./Kamphans, M./Sagebiel, F. (Hrsg.): Subversion und Intervention. Wissenschaft und Geschlechter(un)ordnung, Opladen, S. 139-154

Aulenbacher, B./Riegraf, B. (2010a): The New Entrepreneurship in Science and Changing Gender Arrangements. Approaches and Perspectives, in: Riegraf, B. Aulenbacher, B./Kirsch-Auwärter, E./Müller, U. (Hrsg.): GenderChange in Academia, Wiesbaden, S. 61-74

Aulenbacher, B./Riegraf, B. (2010b): Wissenschaftlerlnnen in der Entrepreneurial University. Über den Wettbewerb der Hochschulen und die Bewegungen in den Geschlechterarrangements, in: Ernst, W. (Hrsg.): Geschlecht und Innovation. Gender-Mainstreaming im Techno-Wissenschaftsbetrieb, Hamburg, S. 42-64 Aulenbacher, B./Riegraf, B. (2011): Die Analyse alltäglicher und biografischer Arbeitsarrangements als Weg arbeits- und industriesoziologischer Sozial- und Zeitdiagnostik, in: Arbeits- und Industriesoziologische Studien (AIS) der Sektion Arbeits- und Industriesoziologie in der DGS 4 (2), Dezember, S. 74-90, www.ais-studien.de

Becker, R./Caspri, A./Kortendiek, B./Münst, S./Schäfer, S. (2010): Gender-Report 2010. Geschlechter(un)gerechtigkeit an nordrhein-westfälischen Hochschulen. Fakten, Analysen, Profile, Essen

Berliner Initiative der Privatdozenten BIP (2012): http://privdoz.de (letzter Zugriff am 16. 03. 2012)

Binner, K. (2010): Is Science as Way of Life in Transition? Some Notes about the Every Day Style of Life of Academics in Germany, in: Riegraf, B./Aulenbacher, B./Kirsch-Auwärter, E./Müller, U. (Hrsg.): GenderChange in Academia, Wiesbaden, S. 149-160

Binner, K./Kubicek, B./Weber, L. (2010): Geschlechterarrangements in den Entrepreneurial Universities. Ein Blick auf Disziplinen, Arbeits- und weitere Lebensverhältnisse, in: Aulenbacher, B./Fleig, A./Riegraf, B. (Hrsg.): Feministische Studien, Schwerpunktheft Organisation, Geschlecht, soziale Ungleichheiten 28 (1), S. 71-84

Bloch, R./Burkhardt, A. (2010): Arbeitsplatz Hochschule und Forschung für wissenschaftliches Personal und Nachwuchskräfte, Hans-Böckler-Stiftung, Arbeitspapier (207), http://www.boeckler.de/pdf/p_arbp_207.pdf

Brugger, P./Buschle, N. (2010): Hochschulen auf einen Blick. Ausgabe 2010, Bundesamt für Statistik, http://www.destatis.de/jetspeed/portal/cms/Sites/ destatis/Internet/DE/ Content/Publikationen/Fachveroeffentlichungen/Bildung ForschungKultur/HochschulenAufEinenBlick,templateld=renderPrint.psm l Buchholz, L. (2004): Wissenschaftskarrieren an österreichischen Universitäten. Erfahrungen und Einstellungen von Professorinnen und Professoren, in: Appelt, E. (Hrsg.): Karriereschere. Geschlechterverhältnisse im österreichischen Wissenschaftsbetrieb, Wien, S. 71-91

Bundesministerium für Bildung, Wissenschaft und Kultur (BMBWK) (2006): Bericht über die Nachwuchsförderung und die Entwicklung der Personalstruktur der Universitäten gem. § 121 (19) UG 2002, http://www.bmwf.gv.at/startseite/mini_menue/service/publikationen/wissenschaft/universitaetswesen/bericht_ueber_die_nachwuchsfoerderung_und_die_entwicklung_der_personalstruktur_der_universitaeten/

Bundesministerium für Frauen und öffentlichen Dienst (BFD) (2010): Frauenbericht 2010. Bericht betreffend die Situation von Frauen in Österreich im Zeitraum von 1998 bis 2008, http://www.frauen.bka.gv.at/site/7207/default.aspx Clark, B. (1998): Creating Entrepreneurial Universities. Organizational Pathways of Transformation, Oxford

Dachverband der Universitäten (DU) (2011): Kollektivvertrag für die MitarbeiterInnen der Universitäten. 01.11.2011. 2. Nachtrag, http://www.tuwien.ac.at/ fileadmin/t/rechtsabt/downloads/Kollektivvertrag/20110215_KV.pdf

Dörre, K./Neis, M. (2008): Forschendes Prekariat? Mögliche Beiträge der Prekarisierungsforschung zur Analyse atypischer Beschäftigungsverhältnisse in der Wissenschaft, in: Klecha, S./Krumbein, W. (Hrsg.): Die Beschäftigungssituation von wissenschaftlichem Nachwuchs, Wiesbaden, S. 127-142 
Enders, J. (2003): Flickwerkkarrieren und Strickleitern in einer prekären Profession. Ein Beitrag zur Nachwuchspolitik an den Hochschulen, in: Hitzler, R./ Pfadenhauer, M. (Hrsg.): Karrierepolitik. Beiträge zur Rekonstruktion erfolgsorientierten Handelns, Opladen, S. 253-262

Esser, H. (2002): Wo steht die Soziologie?, in: Soziologie 31 (4), S. 20-32

Grühn, D./Hecht, H./Rubelt, J./Schmidt, B. (2009): Der wissenschaftliche "Mittelbau" an deutschen Hochschulen. Zwischen Karriereaussichten und Abbruchtendenzen, www.tu-berlin.de/fileadmin/f12/downloads/koop/ publikationen/endbericht_Verdi_Studie_09. pdf.

Haraway, D. (1996): Anspruchsloser Zeuge@Zweites Jahrtausend. FrauMann@ trifft OncoMouse ${ }^{\mathrm{TM}}$, Leviathan und die vier Jots: Die Tatsachen verdrehen, in: Scheich, E. (Hrsg.): Vermittelte Weiblichkeit. Feministische Wissenschafts- und Gesellschaftstheorie, Hamburg, S. 347-389

Hartmann, M. (2010): Die Exzellenzinitiative und ihre Folgen, in: Leviathan 38 (3), S. 369-387

Hellemacher, L. (2011): Gender-Pay-Gaps an Hochschulen, in: die neue hochschule (3), S.122-126

Leontowitsch, M./Váquez-Cupeiro, S. (2003): Above the Glass Ceiling? Preliminary report of Postal Survey of University Professors in the UK, and Merit, Luck, and a good Nanny? Exploring the Intricacies in the CareerTrajectories of Women Academics in Psychology and Engineering, http://csn.uni-muenster. de/women-eu/download/TP\%2003-13\%20Leontowitsch\%20Vasquez-Cupeiro. pdf

Liebig, B. (2010): Academic life and gender relations. The case of fathers in professorship, in: Riegraf, B./Aulenbacher, B./Kirsch-Auwärter, E./Müller, U. (Hrsg.): GenderChange in Academia, Wiesbaden, S. 161-173

Matthies, H. (2005): „Entrepreneurshipping“ in unvollkommenen Märkten das Beispiel Wissenschaft, in: Lohr, K./Nickel, H. M. (Hrsg.): Subjektivierung von Arbeit, Münster, S. 149-179

Metz-Göckel, S. (2009): Diskrete Diskriminierungen und persönliches Glück, in: Riegraf, B./Aulenbacher, B. (Hrsg.): Erkenntnis und Methode. Geschlechterforschung in Zeiten des Umbruchs, Wiesbaden, S.27-49

Metz-Göckel, S./Selent, P./Schürmann R. (2010): Integration und Selektion. Dem Dropout von Wissenschaftlerinnen auf der Spur, in: Beiträge zur Hochschulforschung 32 (1), S. 8-35

Meier, F./Schimank, U. (2004): Neue Steuerungsmuster an den Universitäten. Mögliche Folgen für die geisteswissenschaftliche Forschung, in: Kimmich, D./ Thumfahrt, A. (Hrsg.): Universität ohne Zukunft? Frankfurt a. M., S. 97-123

Meuser, M./Riegraf, B. (2010): Geschlechterforschung und Gleichstellungspolitik. Von der Frauenförderung zum Diversity Management, in: Aulenbacher, B./ Meuser, M./Riegraf, B.: Soziologische Geschlechterforschung. Eine Einführung, Wiesbaden, S. 189-209

Mittelstraß, J. (1982): Wissenschaft als Lebensform. Reden über philosophische Orientierungen in Wissenschaft und Universität, Frankfurt a. M.

Münch, R. (2007): Die akademische Elite. Zur sozialen Konstruktion wissenschaftlicher Exzellenz, Frankfurt a. M.

Münch, R. (2009): Globale Eliten, lokale Autoritäten. Bildung und Wissenschaft unter dem Regime von PISA, McKinsey \& Co, Frankfurt a. M.

Musselin, C (2007): Are Universities Specific Organizations?, in: Krücken, G./ Kosmützky, A./Torka, M. (Hrsg.): Towards a Multiversity? Universities between GlobalTrends and National Traditions, Bielefeld, S. 63-84

Noble, D. F. (1992): A World Without Women. The Christian Clerical Culture of Western Science, New York

Pasternack, P. (2008): Österreich, in: Kreckel, R. (Hrsg.): Zwischen Promotion und Professur, Leipzig, S. 277-301

Rau, A. (2010): Psychopolitik. Macht, Subjekt und Arbeit in der neoliberalen Gesellschaft, Frankfurt a. M.

Riegraf, B. (2007a): Der Staat auf dem Weg zum kundenorientierten Dienstleistungsunternehmen? New Public Management geschlechtsspezifisch analysiert, in: Aulenbacher, B./Funder, M./Jacobsen, H./Völker, S. (Hrsg.): Arbeit und Geschlecht im Umbruch der modernen Gesellschaft, Wiesbaden, S. 78-94

Riegraf, B. (2007b): New Public Management und Geschlechtergerechtigkeit. Sozialer Fortschritt 56 (9-10), S. 259-263

Riegraf, B. (2009): Die Organisation von Wandel. Gender-Wissen und GenderKompetenz in Wissenschaft und Politik, in: Riegraf, B./Plöger, Lydia (Hrsg.): Gefühlte Nähe - faktische Distanz. Geschlecht zwischen Wissenschaft und Politik, Opladen, S. 67-80
Sambale, J./Eick, V./Walk, H. (2008): Das Elend der Universitäten: Neoliberalisierung deutscher Hochschulpolitik, Münster

Schimank, U. (2005): Die akademische Profession und die Universitäten: New Public Management und eine drohende Entprofessionalisierung, in: Klatetzki, T./ Tacke, V. (Hrsg.): Organisation und Profession, Wiesbaden, S.143-164

Simons, K./Hellemacher, L. (2009): W-Zulagen und Entgeltgleichheit an Hochschulen, in: die neue hochschule (4-5), S. 14-15

Statistik Austria (2011): Bildung in Zahlen 2009/10. Tabellenband. Bundesanstalt Statistik Österreich, Wien

Statistisches Bundesamt (2011): Personal an Hochschulen 2010. Bildung und Kultur, Fachserie 11, Reihe 4.4, Wiesbaden, www.destatis.de

Teichler, U. (2008): Der Jargon der Nützlichkeit. Zur Employability-Diskussion im Bologna-Prozess, in: Das Hochschulwesen. Forum für Hochschulforschung, -praxis und -politik 56 (3), S. $68-79$

Thomas, R./Davies, A. (2002): Gender and New Public Management: Reconstituting Academic Subjectivities, in: Gender, Work and Organization 9 (4), S. 372-397

Weber, M. (1994 [1922]): Wissenschaft als Beruf. Studienausgabe (MWS) Bd. I/17, Tübingen

Wetterer, A. (2002): Arbeitsteilung und Geschlechterkonstruktion, Konstanz Zimmer, A./Krimmer, H./Stallmann, F. (2007): Frauen an Hochschulen. Winners among losers. Zur Feminisierung der deutschen Universität, Opladen

\section{AUTORINNEN}

Brigitte Aulenbacher, Dr., Professorin für Soziologische Theorie und SozialanaIysen am Institut für Soziologie an der Johannes Kepler Universität Linz. Arbeitsschwerpunkte: Arbeits-, Organisationssoziologie, Geschlechterforschung, Gesellschaftstheorie.

brigitte.aulenbacher@jku.at

Kristina Binner, Sozialwissenschaftlerin, wissenschaftliche Mitarbeiterin am Institut für Soziologie der Johannes Kepler Universität Linz. Arbeitsschwerpunkte: SoziologischeTheorie, Geschlechter- und Hochschulforschung.

kristina.binner@jku.at

Birgit Riegraf, Dr., Professorin für Allgemeine Soziologie an der Fakultät für Kulturwissenschaften der Universität Paderborn. Arbeitsschwerpunkte: Gesellschafts- und Gerechtigkeitstheorien, Arbeits- und Organisationssoziologie, Geschlechterforschung.

briegraf@mail.upb.de

Lena Weber, Soziologin, wissenschaftliche Mitarbeiterin an der Fakultät für Kulturwissenschaften der Universität Paderborn. Arbeitsschwerpunkte: Theoretische Soziologie, Organisationssoziologie, Geschlechter- und Wissenschaftsforschung.

leweber@mail.upb.de 